\title{
Perspective on Robotic Assisted Gait Training Devices
}

\section{Ross Bogey. D.0*}

Director, Physical Medicine and Rehabilitation Residency Program, Casa Colina Hospital and Centers for Healthcare, USA

Received: 此 May 01, 2018; Published: 㘹 May 07, 2018

*Corresponding author: Ross Bogey. D.O, Director, Physical Medicine and Rehabilitation Residency Program, Casa Colina Hospital and Centers for Healthcare, 255 East Bonita Avenue, Pomona, CA 91769, USA, Email: rossbogey@gmail.com

\section{Perspective}

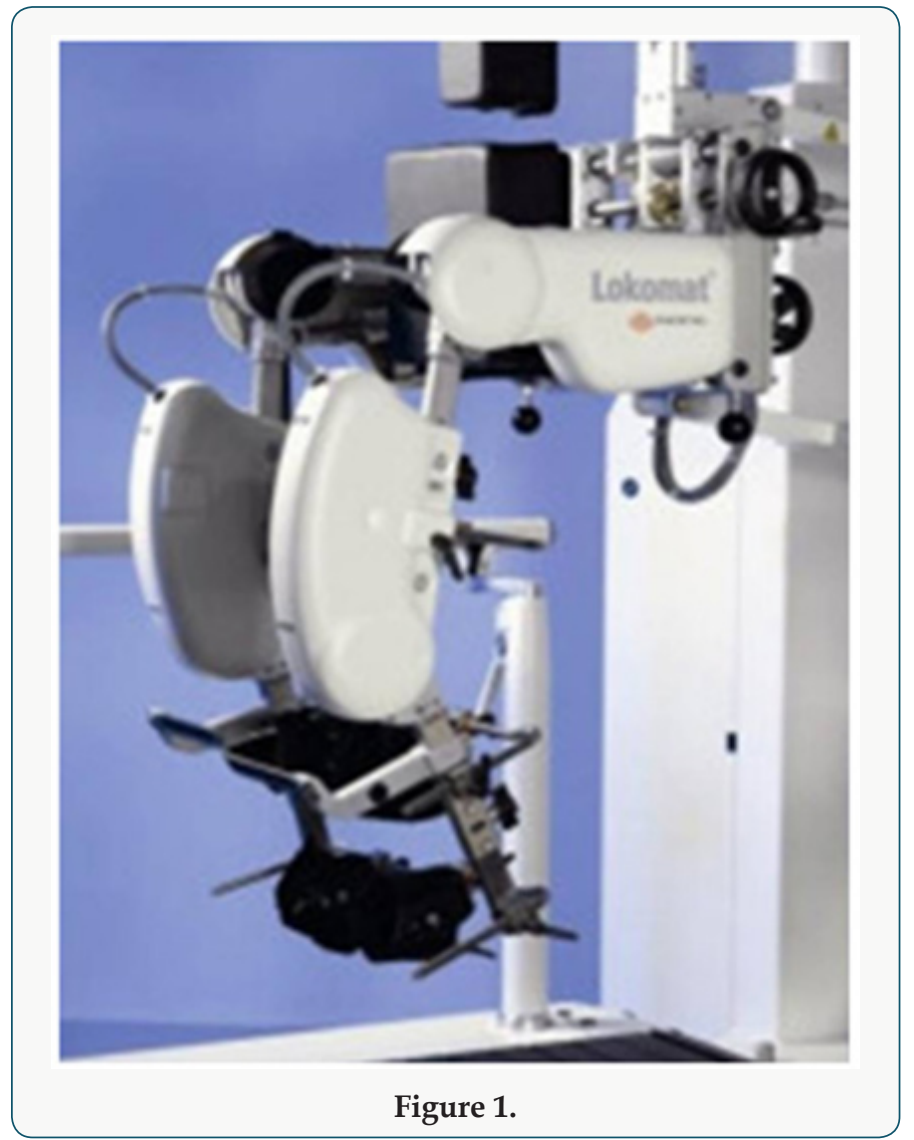

Adults with neurological injury (e.g. stroke, spinal cord injury) report that recovery of walking is a primary goal [1]. While many recover at least the ability to perform short distance, household level walking, community level ambulation at even moderate walking speeds is beyond the ability of most of these individual [2]. While the brain is involved in several aspects of gait initiation, termination, responding to environmental perturbations much of walking is controlled and monitored in the spinal cord, specifically in the central pattern generators (CPG). This was demonstrated nearly one half century ago, where early studies of spinal cord injured quadrupeds demonstrated reciprocal locomotion patterns in the absence of direct signals from the brain. Early studies of spinal cord injured cat [3] demonstrated that gait recovery was possible when the effects of gravity were attenuated. Gait recovery in neurologically impaired humans has also been shown using the same paradigms [4]. Good outcomes, as measured by walking speed, distance walked per time ( 6 minute walk test), and improved cardiac response have been shown in BWSTT trials in persons with stroke [5].

While outcomes with BWSTT are generally favorable, the approach is labor-intensive and may require more than one therapist per patient during gait recovery sessions [6]. At about the time of the turn of this century an alternative approach was advanced. In lieu of using multiple physical therapists and to limit the physical demands on those therapists robotic devices were developed that would essentially perform the physical work provided by the therapists [7]. In addition to reducing the number of therapists required for each training session, an associated factor favoring this approach was that more uniform therapy could be provided by using robotic assisted training, and results more easily quantified. That is, the robotic devices could be programmed in theory to provide a desirable amount of assistance a priori, and that gait recovery outcomes would also be more predictable.

Unfortunately, the real world observed outcomes with robotic assisted gait training have not met the aforementioned expectations [6]. The robotic design(s) and the motor learning paradigms used have each contributed to the sub-optimal results seen to date. Early robotic assisted gait training devices did not have back-drivability as a key design element. The exoskeleton generally used (e.g Lokomat) would take the patient through the walking cycle with pre-determined kinematic patterns at the lower extremity joints (Figure 1). Presumably the device user would ingrain this pattern then continue to walk in a similar matter when not constrained by the robotic assisted device. Yet this approach is not supported by the motor learning literature, and has not led to improved walking for the most part [6]. In direct comparisons where factors were matched except for the use/non-use of robotic assisted devices in tread mill training those patients who did not get robotic assistance had higher walking speeds and improved cardiac training as compared to cohorts that received robotic assisted therapy. 
Current robotic-assisted devices have substantial shortcomings. One factor that curbs desirable outcomes with these devices is the relatively slow walking speeds that can be used during therapy sessions with the devices. A further, and likely more important design limitation, is the number and placement of actuators used. Early devices had actuators were placed at the bilateral hips and bilateral knees, to provide assistance as needed. This trend continues, with no robotic-assisted devices (at the time this goes to press) effectively addressing shortcomings in either plantar flexion or dorsiflexion for device users. This is unfortunate for two key reasons. First, ankle plantar flexion power [7] is substantially higher than hip extensors or hip adductors [9] (Figure 2). While not shown, hip abductors and hip flexors produces even less power during level adult walking [9]. Further, the more distal muscles specifically the plantar flexors and dorsiflexors are typically more impaired than more proximally located muscles after CNS injury such as stroke. To date, the "solution" has been to apply a device at the ankle which keeps it essentially in a neutral posture throughout the gait cycle (See Figure 1). Clearly the recovery of effective ankle motion (plus force and power) is not possible via this approach.

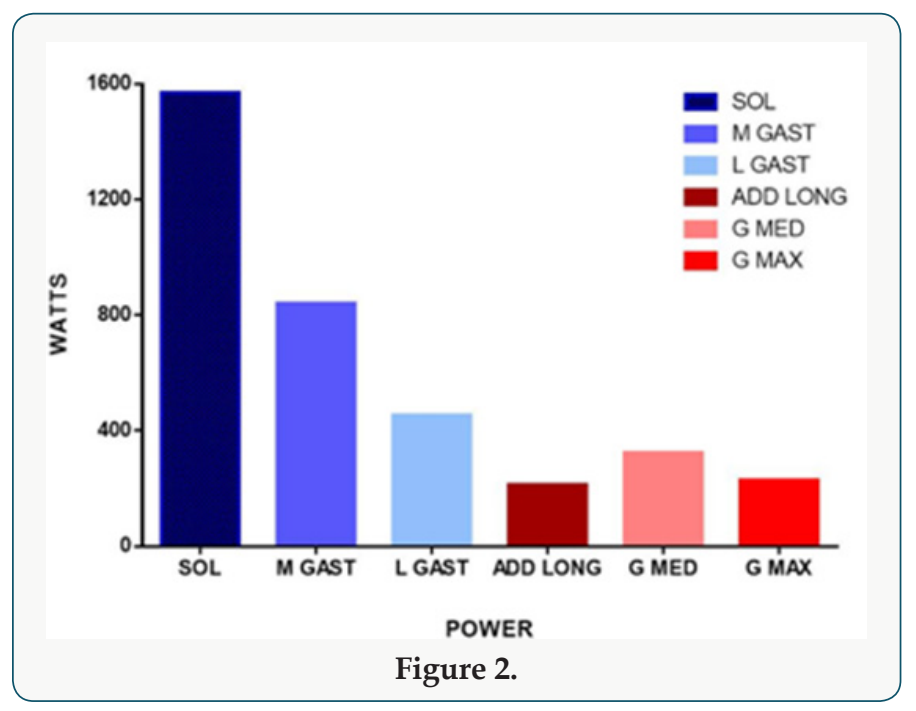

It is unlikely that the importance of the more distally located muscles is lost on robotics engineers. More likely, they have pursued more "low hanging fruit" in the first and second generation devices. It is relatively easy to place actuators at the hip, where their actuator mass does not confound the desired walking cycle.
However, placing a similarly-sized actuator at the ankle joint is a daunting engineering problem. Here the swinging mass of the actuator plus foot must be controlled such that swing phase kinematics is acceptable. To date the "solution" has been to fix that ankle through a very limited joint range (holding it in a relatively neutral position) and ignore recovery of ankle motion as part of gait training sessions. Most rehabilitation specialists believe that robotic assisted movement recovery will be a part of the therapy landscape in short order [10]. However, the currently available devices need to overcome, at minimum, the design flaws listed above. Improved walking by our patients and improved rehabilitation outcomes overall hang in the balance.

\section{References}

1. Mansfield A, Wong JS, Bayley M, Blasin L, Brooks D, et al. (2013) Using wireless technology in clinical practice: Does feedback of daily walking activity improve walking outcomes of individuals receiving rehabilitation post stroke? BMC Neurol 13: 93.

2. Bogey RA, Hornby TG (2007) Gait training strategies utilized in poststroke rehabilitation: Are we really making a difference? Topics Stroke Rehabil 14(6): 1-8.

3. Smith JL, Edgerton VR, Elred E, Zernicke RF (1983) The chronic spinalized cat: A model for neuromuscular plasticity. Birth Defects 19(4): 357-373.

4. Hesse S, Uhlenbrock D, Sarkodie Gyan T (1999) Gait pattern of severely disabled hemiparetic subjects on a new controlled gait trainer as compared to assisted treadmill walking with partial body weight support. Clin Rehabil 13(5): 401-410.

5. Macko RF, Smith GV, Dobrovolny CL, Sorkin JD, Goldberg AP, et al. (2001) Treadmill training improves fitness reserve in chronic stroke patients. Arch Phys Med Rehabil 82(7): 879-884.

6. Hidler J, Nichols D, Pelliccio M, Brady K, Campbell DD, et al. (2009) Multi center randomized clinical trial evaluating the effectiveness of the Lokomat in sub acute stroke. Neurorehabil Neural Rep 23(1): 5-13.

7. Hornby TG, Campbell DD, Kahn JH, Bernott T, Moore JL, et al. (2008) Enhanced gait related improvements after therapist versus robotic assisted locomotor training in subjects with chronic stroke: A randomized controlled trial Stroke 39(6): 1786-1792.

8. Bogey RA, Gitter AJ, Barnes LA (2010) Determination of ankle muscle power in normal gait using an EMG to force processing approach. J Electromyogr Kinesiol 20(1): 46-54.

9. Bogey RA, Barnes LA (2017) Estimates of individual muscle power production in normal adult walking. J Neuroeng Rehabil 11: 14-20.

10. Krebs HI (2013) Integrating robot assisted interventions into standard of care. NeuroRehabil 33: 1-2. 
(c) (P) This work is licensed under Creative To Submit Your Article Click Here: Submit Article

DOI: $10.32474 /$ OAJBEB.2018.02.000136



